Accepted Article (Journal: Drug, Alcohol \& Dependence)

\title{
Alcohol And Disgust:
}

\section{An Intimate Relationship}

Lorenzo D. Stafford, Alistair Sekulla, Edward Morrison, Diana S.

Fleischman, Alistair J. Harvey

Centre for Comparative and Evolutionary Psychology

Department of Psychology, University of Portsmouth, U.K.

Correspondence to be sent to: Lorenzo D. Stafford, Department of Psychology, University of Portsmouth, King Henry Building, King Henry I Street, Portsmouth PO1 2DY.U.K. Email: lorenzo.Stafford@port.ac.uk. Tel: 02392846322. Fax: 02392846300

Conflict of Interest: All authors declare they have no conflict of interest. 


\begin{abstract}
Background: Alcohol intoxication has been associated with increases in risk taking behavior and more ambiguously, alterations in emotional perception. In the first study of its kind, we examine how theories of disgust can be used to help explain these effects.

Methods: Using a single-blind procedure, participants $(\mathrm{n}=73)$ were randomly allocated to an alcohol (Males: 0.68g/kg; Females: $0.60 \mathrm{~g} / \mathrm{kg}$ ) or placebo condition and then completed a psychometric measure of disgust (TDDS).

Results: Results revealed a non-significant trend toward lower disgust sensitivity in the alcohol versus placebo condition. We did however find a significant negative correlation, whereby increases in breath alcohol level were associated with decreased pathogen disgust.

Conclusions: These findings demonstrate a relationship between breath alcohol level and disgust sensitivity which could help explain differences in risk associated behavior.
\end{abstract}

\title{
Keywords
}

Alcohol, Disgust, Emotions 


\subsection{Introduction}

Alcohol is a key factor in the aetiology of many diseases and injuries (WHO 2014) and is associated with increases in risky behaviour (Foster and Ferguson, 2012). More recently, research has examined the effects of alcohol administration on the processing of social-emotional cues. Since alcohol is frequently consumed in social situations, being able to identify the correct facial emotion in others (e.g. fear rather than anger) would have obvious advantages in deescalating potentially violent situations (Marsh et al., 2007; Attwood and Munafo, 2014). Research in this area has shown mixed evidence, with some work reporting alcohol impairments to emotional facial recognition (Tcherkassof et al., 2011) but in contrast no effects reported elsewhere (Walter et al., 2011). Of particular interest here, one recent study found that alcohol administration resulted in enhanced perception of disgust (and contempt) (Felisberti and Terry, 2015). In order to explain the disgust related findings, the authors theorise that alcohol may exert an immunosuppressant effect (Szabo and Saha, 2015), thereby increasing the risk of disease which is then mitigated by an increase in disgust sensitivity to help combat that risk. This theory is broadly consistent with related research where increases in Perceived Vulnerability to Disease (PVD) have been associated with increases in disgust sensitivity (Duncan et al., 2015, see also Fleischman and Fessler 2018). However, since the focus of that study (Felisberti and Terry, 2015) was facial response accuracy, it is unclear from that work alone whether 'sensitivity' to disgust had actually changed. In the wider domain of disgust research, disgust sensitivity is measured using various validated questionnaires, one of which is the Three Domains of Disgust Scale (TDDS, Tybur et al., 2009). This questionnaire was developed from the theory that disgust evolved as a 'behavioural' immune system to help guard against certain environmental threats, categorized as pathogen (e.g. rotten food, faeces, disease), sexual (sexually transmitted disease) 
and moral disgust (moral transgressions) (Tybur et al., 2009). Work has shown how increases in state disgust are associated with increasing preoccupation toward health/cleaning concepts (Jones and Fitness, 2008) and preventative health strategies (Tybur et al., 2011). From the opposite perspective, research has also demonstrated how following a sexual arousal induction, ratings toward 'sexual' disgust elicitors (visual, auditory, tactile) were lower compared to those in control conditions (Stevenson et al., 2011). The theory used to explain this finding is based on the premise that some aspects of sexual behaviour may appear as high in disgust (i.e. as an increased risk of disease) in a non-sexually aroused state but when an individual is sexually aroused, necessarily, disgust is downregulated to facilitate sex. Similar effects have been observed where individuals in a high hunger state exhibited lower facial disgust activity ${ }^{1}$ when exposed to pictures of unpalatable food (Hoefling et al., 2009). Collectively, these studies suggest that alterations in drive states (sexual arousal, hunger) can reduce disgust (sexual, pathogen) sensitivity and could potentially lead to more risk taking (having unprotected sex, consuming spoiled food). However, what is unknown is the relationship between alcohol and disgust sensitivity, which is important to understand as alcohol intoxication is often paired with a number of these potential disgust risks (e.g., sex, poorer hygiene, moral transgressions/crime)

We examine this question in the present study, where individuals consumed a moderate dose of alcohol or a placebo beverage and then completed a measure of disgust sensitivity (TDDS, Tybur et al., 2009). Given the higher incidences of risk taking behaviour that include risky sexual behaviour and unprotected sex (Engineer et al., 2003; Rehm et al., 2012) associated with general alcohol consumption, one would predict lower disgust sensitivity in response to

\footnotetext{
${ }^{1}$ Facial regions (levator labii superioris) that control characteristic disgust response were lower in the food deprived state
} 
alcohol. In contrast, if the immunosuppressant theory is correct (Felisberti and Terry, 2015), we would expect alcohol to increase disgust sensitivity. The study also aimed to analyse changes in the domain of disgust, which we tentatively predict would be stronger for pathogen and sexual disgust.

\subsection{Material and methods}

\subsection{Participants}

Seventy-three (52 female) University students, aged between 18 and 54 years $(M=23.0$, $S D=6.4)$ participated in this study. Participants were recruited following the completion of a different study looking at the effects of alcohol on eyewitness identification. For that study, only regular consumers of alcohol were recruited (consuming between 5-50 UK alcohol units per week), who were not alcohol dependent (defined by the Michigan Alcohol Screening Test, MAST). Females who were (or thought they might be) pregnant were excluded from the study. Upon completion of the identification experiment they were offered the opportunity to take part in the current study, which was described as 'Understanding factors associated with alcohol consumption’. Participants were compensated with course credit or a $£ 10$ honorarium. The sample size was based partly on the requirements of that (eyewitness identification study) but also on research more closely related to the present study. The study protocol was given ethical approval from the University's ethics committee (British Psychology Society guidelines, similar to the Declaration of Helsinki).

\subsection{Design}


A between-subjects design was used, where participants were randomly allocated to one of two groups (alcohol/placebo). The breath alcohol concentration (BrAC) and disgust sensitivity scores for the Three Domains of Disgust Scale (TDDS) were the dependent variables.

\subsection{Materials}

All participants completed the Three Domains of Disgust Scale, a 21 item questionnaire (TDDS; Tybur et al., 2009). The TDDS measures individual sensitivity to pathogen, sexual and moral disgust as separate domains. The scale consists of 21 items -7 for each domain - for which participants responded on a 7-point Likert-like scale ranging from 'not at all disgusting' to 'extremely disgusting'. Examples of pathogen disgust items were "Stepping on dog poo" and "Standing close to a person who has body odour"; examples of sexual disgust were "Hearing two strangers having sex" and "Bringing someone you just met back to your room to have sex"; and examples of moral disgust were "Stealing from a neighbour" and "Forging someone's signature on a legal document". Some words were adapted to be more applicable to English society - such as 'poop' to 'poo'. Scores for each domain ranged from 0-6, and were the sum of scores for the particular domain's items. Higher scores pertained to higher disgust sensitivity. The TDDS has been shown to have high levels of reliability, Cronbach's alpha for each factor (pathogen: .83, sexual: .86, moral: .89, Tybur et al., 2009).

\subsection{Drinks and administration}

Drinks were administered single-blind and were freshly prepared for each participant at a dose of $1.7 \mathrm{ml}$ vodka (Absolut, $40 \%$ alcohol-by-volume) $/ \mathrm{kg}$ (males) and $1.5 \mathrm{ml}$ vodka $/ \mathrm{kg}^{2}$

\footnotetext{
${ }^{2}$ Equivalent in alcohol: Males: $0.68 \mathrm{~g} / \mathrm{kg}$; Females: $0.60 \mathrm{~g} / \mathrm{kg}$
} 
(females), mixed with enough sugar free Indian tonic water to produce a 500ml drink. Hence, a female weighing $70 \mathrm{~kg}$ would be given $105 \mathrm{ml}(\approx 4 \mathrm{UK}$ alcohol units) of vodka ( $40 \% \mathrm{ABV}) . \mathrm{We}$ used a slightly lower dose for females since there is evidence that they experience greater impairments than males when receiving the same alcohol dose (e.g., Mumenthaler et al., 1999). These alcohol doses were selected to produce a BAC up to or just over the legal driving limit for England (i.e., BAC $=>0.08 \%$ ). The placebo drink consisted of 500ml Indian tonic water (Tesco low calorie sugar free) with traces of vodka dropped on to the surface (3-4 drops) and mistsprayed over the glass to create a strong alcohol odour. This was completed to disguise the fact that participants had been given a non-alcoholic beverage thereby controlling alcohol expectancies across treatment groups.

\subsection{Procedure}

All testing took place in test rooms at the psychology department of the University. Participants were instructed not to consume alcohol 24-hours before testing and to avoid eating 4-hours before their session. On arrival at the laboratory, participants were breath tested (Dräeger Alcotest 6510 breathalyser), to verify that their BrAC was zero, and weighed to calculate the alcohol dose to be administered. They were then presented with their beverage (alcohol/placebo) and given 20 minutes to consume the drink, followed by a 30-minute alcohol absorption period. Next, participants rinsed their mouths with water to remove residual traces of alcohol and gave a second BrAC reading. They then completed cognitive tests as part of that (eyewitness identification) study (appx 15mins). Participants who agreed to take part in the present study gave their informed consent and then completed the TDDS. They were then given a full debriefing which included information on their group (alcohol/placebo) allocation. Those 
participants in the alcohol condition were advised to stay in the waiting area until their $\mathrm{BrAC}$ level was less than $0.18 \mathrm{mg} / \mathrm{l}(<0.04 \%$ BAC); alternatively, they could leave after signing a disclaimer form to say they acknowledged being made aware of their level of intoxication and of being advised not to drive, ride a bicycle or operate machinery.

\subsection{Data Analyses}

The data were screened for outliers ( \pm 2 sd from group mean), with any resulting scores replaced with the mean of that group. A 3x2x2 Multivariate General Linear Model was completed with each of the three domains of disgust as dependent variables, and Condition (alcohol vs placebo) and Sex (male vs female) as independent variables.

\subsection{Results}

For pathogen and sexual disgust, analyses revealed a trend toward lower scores in the alcohol versus placebo condition but these did not reach significance: pathogen, $F(1,69)=1.91$, $p=.17$, sexual, $F(1,69)=1.70, p=.20$; with little difference in moral disgust, $F(1,69)=.11, p=$ .74 (Table 1). There was a main effect of Gender on sexual disgust, $F(1,69)=7.69, p=.007, \eta^{2}$ $=.10$, with higher scores in females $(M=18.0, S E=1.1)$ versus males $(M=12.5, S E=1.7)$. None of the Condition x Gender effects were significant (all Fs <0.6).

To further understand the relationship between alcohol and disgust, we completed separate bivariate correlations between breath alcohol and pathogen and sexual disgust ${ }^{3}$, for participants in the alcohol condition. For pathogen disgust only, this revealed a significant

\footnotetext{
${ }^{3}$ We did not include Moral disgust as there was no difference in the mean values between the alcohol/placebo group
} 
negative association, $(r=-0.35, \mathrm{n}=38, p=.032)$, hence increases in breath alcohol predicted lower pathogen disgust scores (Figure 1).

\subsection{Discussion}

The study found no significant difference in disgust sensitivity between the alcohol and placebo groups, which was against our prediction. However, we did find a negative association between breath alcohol and pathogen disgust which (to our knowledge) constitutes the first demonstration of a relation between alcohol level and disgust sensitivity. The finding that breath alcohol was related to pathogen but not sexual disgust was in a sense surprising, given the relation between alcohol and sexual behavior (Engineer et al., 2003; Rehm et al., 2012). Previous work had also shown that increases in sexual arousal led to a lower perception of disgust (Stevenson et al., 2011) which was theorized to be linked to a reduced sensitivity to disgust to permit sexual intercourse. Taken together, these lines of evidence would lead us to expect alcohol ingestion to reduce sexual disgust. However, the differences in methodology between the studies need to be considered, including the fact that, in the Stevenson et al. (2011) study, sexual arousal rather than alcohol level was manipulated. Hence, although sexual arousal is often experienced with alcohol consumption, it may be the case that alcohol taken in isolation is not sufficient to reduce sexual disgust. In contrast, our findings may demonstrate an exclusive association between alcohol and pathogen disgust sensitivity, which would suggest alcohol moderates our concerns about contaminating microorganisms and thus avoidant responses to unsafe/spoiled food and other harmful pathogen sources. These findings are in broad agreement with related work where manipulation of hunger state led to decreased disgust responses to spoiled food (Hoefling et al., 2009) and suggest the separate manipulation of hunger and alcohol can influence disgust perception. At first glance, this may seem surprising, however, it is worth 
reflecting that in many social drinkers and across cultures, alcohol is often consumed prior to food consumption as an aperitif, which can then lead to subsequent increases in hunger (the 'appetiser' effect), so alcohol may indirectly reduce disgust sensitivity by stimulating hunger. Additionally, for many (especially young) drinkers, alcohol consumption continues in the absence of a meal and thereby, we assume, reduce disgust sensitivity even more dramatically in binge drinking contexts. Beyond this anecdotal evidence, research has shown that compared to placebo controls, alcohol increases food consumption (Kwok et al., 2019) and preference for unhealthy snack foods in particular (Rose et al., 2005). However, since we did not measure hunger state in this study, our suggestion that alcohol reduces disgust sensitivity through increases in hunger must remain a speculation.

Alcohol is one of the most widely abused recreational drugs (Peters et al., 2016) and is heavily embedded in many cultures and customs (Gordon et al., 2012). Alcohol's usage in many social contexts is particularly relevant here, in that its intoxicating effects appear to interact with our natural defence system. The initial work that inspired the present study found evidence for alcohol to specifically enhance facial disgust perception (Felisberti and Terry, 2015), which raised the possibility that alcohol may enhance sensitivity to disgust cues, possibly linked to its immunosuppressant effects (Szabo and Saha, 2015). The findings from the present study do not appear consistent with those theoretical predictions but instead suggest that alcohol acts to 'modulate' the emotion of disgust and specifically reduce its sensitivity. To our knowledge, this is the first study to establish a link between alcohol administration and disgust sensitivity and provides a useful framework between applied and theoretical areas of alcohol research. 


\section{References}

Attwood, A. S., and Munafò, M. R., 2014. Effects of acute alcohol consumption and processing of emotion in faces: implications for understanding alcohol-related aggression. J.

Psychopharmacol. 28, 719-732.

DeBruine, L. M., Jones, B. C., Tybur, J. M., Lieberman, D., and Griskevicius, V., 2010. Women's preferences for masculinity in male faces are predicted by pathogen disgust, but not by moral or sexual disgust. Evol. Hum. Behav. 31, 69-74.

Duncan, L. A., Schaller, M., and Park, J. H., 2009. Perceived vulnerability to disease:

Development and validation of a 15-item self-report instrument. Pers. Indiv. Differ. 47, 541546.

Engineer, R., Phillips, A., Thompson, J., and Nicholls, J., 2003. Drunk and disorderly: a qualitative study of binge drinking among 18-to 24-year-olds. London: Home Office.

Felisberti, F., and Terry, P., 2015. The effects of alcohol on the recognition of facial expressions and microexpressions of emotion: enhanced recognition of disgust and contempt. Hum. Psychopharm. Clin. 30, 384-392.

Fleischman, D. S., and Fessler, D. M., 2018. Response to "Hormonal correlates of pathogen disgust: Testing the compensatory prophylaxis hypothesis”. Evol. Hum. Behav. 39, 468-469.

Foster, J.H. and Ferguson, C., 2013. Alcohol 'pre-loading': a review of the literature. Alcohol. Alcoholism. 49, 213-226.

Gordon, R., Heim, D. and MacAskill, S., 2012. Rethinking drinking cultures: A review of drinking cultures and a reconstructed dimensional approach. Public. Health. 126, 3-11.

Jones, B. C., Fincher, C. L., Welling, L. L., Little, A. C., Feinberg, D. R., Watkins, C. D., AlDujaili, E.A. and DeBruine, L.M., 2013. Salivary cortisol and pathogen disgust predict men's preferences for feminine shape cues in women's faces. Biol. Psychol. 92, 233-240.

Kwok, A., Dordevic, A.L., Paton, G., Page, M.J. and Truby, H., 2019. Effect of alcohol consumption on food energy intake: a systematic review and meta-analysis. Brit J Nutr, 121, 481-495.

Marsh, A. A., Kozak, M. N., and Ambady, N., 2007. Accurate identification of fear facial expressions predicts prosocial behavior. Emotion. 7, 239-251.

Mumenthaler, M. S., Taylor, J. L., O'Hara, R., and Yesavage, J. A., 1999. Gender differences in moderate drinking effects. Alcohol. Res. Health. 23, 55-64. 
Peters, S.T., Bowen, M.T., Bohrer, K., McGregor, I.S. and Neumann, I.D., 2017. Oxytocin inhibits ethanol consumption and ethanol-induced dopamine release in the nucleus accumbens. Addict. Biol. 22, 702-711.

Rehm, J., Shield, K. D., Joharchi, N., and Shuper, P. A., 2012. Alcohol consumption and the intention to engage in unprotected sex: Systematic review and meta-analysis of experimental studies. Addiction. 107, 51-59.

Rose, A.K., Hardman, C.A. and Christiansen, P., 2015. The effects of a priming dose of alcohol and drinking environment on snack food intake. Appetite, 95.341-348.

Stevenson, R.J., Case, T.I. and Oaten, M.J., 2011. Effect of self-reported sexual arousal on responses to sex-related and non-sex-related disgust cues. Arch. Sex. Behav. 40, 79-85.

Szabo, G., and Saha, B., 2015. Alcohol's effect on host defense. Alcohol. Res-Curr. Rev. 37, 159.

Tcherkassof, A., Mandran, N., Dubois, M., and Begue, L., 2011. The effects of acute alcohol drinking on the judgment of spontaneous and dynamic facial expressions of emotions. Psychol. Fr. 56, 189-202.

Tybur, J. M., Lieberman, D., and Griskevicius, V., 2009. Microbes, mating, and morality: individual differences in three functional domains of disgust. J. Pers. Soc. Psychol. 97, 103.

Walter, N. T., Mutic, S., Markett, S., Montag, C., Klein, A. M., and Reuter, M., 2011. The influence of alcohol intake and alcohol expectations on the recognition of emotions. Alcohol. Alcoholism. 46, 680-685.

World Health Organization, 2014. Alcohol. Retrieved from http://www.who.int/mediacentre/factsheets/fs349/en/ 
Table 1

Descriptive statistics for the placebo and alcohol groups. Data are mean (SE) unless otherwise stated.

\begin{tabular}{|c|c|c|c|c|}
\hline & \multicolumn{4}{|c|}{ Group } \\
\hline & \multicolumn{2}{|c|}{ Placebo $(n=35)$} & \multicolumn{2}{|c|}{ Alcohol $(n=38)$} \\
\hline & $\mathbf{M}$ & SE & $\mathbf{M}$ & SE \\
\hline Age & 22.5 & .06 & $23.3(1.3)$ & 1.3 \\
\hline Gender (F/M) & $35 / 12$ & - & $38 / 10$ & - \\
\hline \multicolumn{5}{|l|}{ Breath Alcohol } \\
\hline$(\mathrm{mg} / \mathrm{L})$ & $\mathrm{n} / \mathrm{a}$ & - & $0.29(.06)$ & .06 \\
\hline TDD-Pathogen & 24.3 & 1.3 & $21.6(1.3)$ & 1.3 \\
\hline TDD-Sexual & 16.6 & 1.4 & $13.9(1.4)$ & 1.4 \\
\hline TDD-Moral & 27.1 & 1.3 & $27.7(1.3)$ & 1.3 \\
\hline
\end{tabular}




\section{Legend:}

Figure 1: Scatterplot Of Pathogen Disgust Scores And Breath Alcohol Reading 


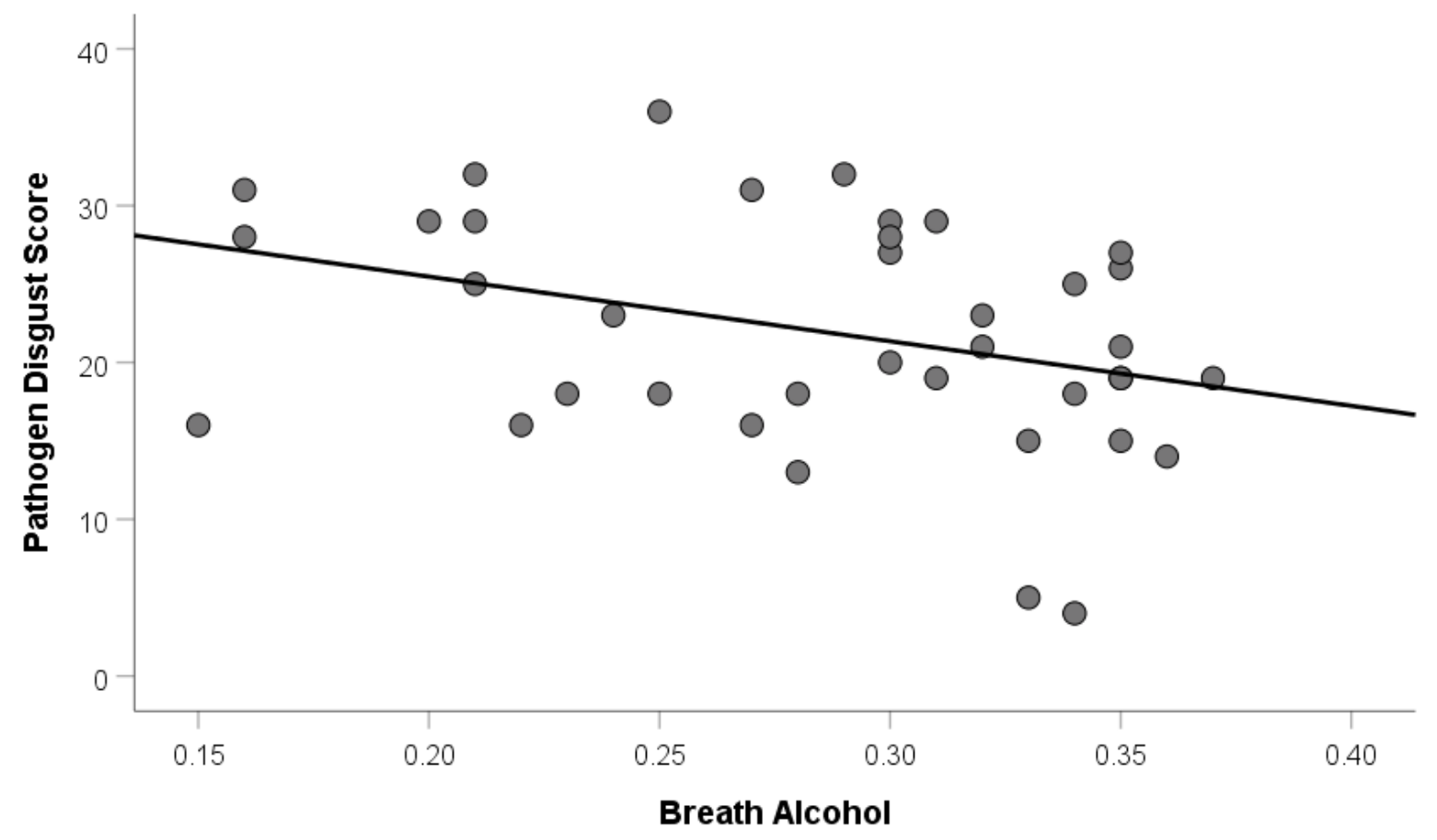

$-15-$ 